
\title{
R Research Square \\ Elevated levels of miR-483-5p in oocytes aggravates premature ovarian insufficiency induced by CDDP by targeting the FKBP4
}

huihui zhao ( $\sim$ huihuiz2020@163.com )

Southern Medical University Nanfang Hospital https://orcid.org/0000-0002-7607-3527

\section{Wenqing Gu}

Sun Yat-Sen Memorial University

Huogui Ouyang

Zhangmuotu hospital of Dongguan

\section{Wenbin Pan}

southern medical university

\section{Hanbin Zhang}

southern medical university

\section{Ling Shuai}

southern medical university

\section{Ruiying Diao}

southern medical university

\section{Liping Wang}

southern medical university

\section{Research}

Keywords: Premature ovarian insufficiency, CDDP, ovarian injury, miR-483-5p, FKBP4

Posted Date: August 4th, 2020

DOl: https://doi.org/10.21203/rs.3.rs-17131/v3

License: (1) (1) This work is licensed under a Creative Commons Attribution 4.0 International License. Read Full License 


\section{Abstract}

Background: Premature ovarian insufficiency $(\mathrm{POI})$ is characterized by a loss of ovarian function before 40 years-of-age and represents an existing challenge cause of female infertility. POI is one of the dominant causes of cis-diaminedichloroplatinum (cisplatin, CDDP)-induced reproductive impairment. However, the detailed mechanisms underlying POI induced by CDDP remain unclear.

Methods: The POI C57B6/J mouse model was created by administering CDDP. The effects of FKBP4 were investigated using isobaric tags for relative and absolute quantification analysis (iTRAQ), real-time quantitative PCR (qRT-PCR) and western blotting. Target prediction was predicted using TargetScan software. Levels of sex hormones were tested using Enzyme-linked immunosorbent assays (ELISA).

Results: We found that the FKBP4 protein was down-regulated in the ovaries of CDDP model. Target prediction identified FKBP4 as a potential target for miR-483-5p, which was expressed at high levels in both the ovaries and serum of CDDP-POI mice, and in the serum from POI patients. In vitro experiments further confirmed that FKBP4 was the target for miR-483-5p in human cervical cancer cells (HeLa). The overexpression of FKBP4 in human granulosa cells (KGN) alleviated the apoptosis caused by CDDP and the overexpression of miR-483-5p. Furthermore, the overexpression of miR-483-5p in oocytes caused injury to the ovaries, and disrupted the levels of sex hormones in CDDP-POI mice (AMH: $P<0.01 ; E_{2}: P<$ 0.01 ; FSH: $P<0.01)$.

Conclusions: Analyses showed that miR-483-5p targets the FKBP4 protein in a mouse model of POI induced by CDDP. Elevated levels of miR-483-5p in oocytes could aggravate POI induced by CDDP by targeting FKBP4. Overall, our data demonstrate that miR-483-5p was responsible for the underlying pathophysiology of POI induced by chemotherapeutic treatments, such as CDDP.

\section{Background}

Premature ovarian insufficiency (POI), is one form of the female infertility, defined as amenorrhea (primary or secondary) for at least 4 months with elevated levels of follicle-stimulating hormone (FSH) $>25 \mathrm{IU} / \mathrm{L}$ on two occasions $>4$ weeks apart, prior to 40 years-of-age[1, 2]. It affects approximately $1 \%$ women by age 40 years and $0.1 \%$ by age 30[3]. The etiology of POI is heterogeneous and multifactorial, $90 \%$ of $\mathrm{POI}$ cases are idiopathic, as a result of genetic, enzymatic, autoimmune, infection and iatrogenic, such as chemotherapy[4]. Chemotherapy have radically increased long-term survival of young cancer patients, however, major side effects are premature ovarian insufficiency and infertility[5]. Cisdiaminedichloroplatinum (cisplatin, CDDP) is a first-line chemotherapeutic drug that is widely used to treat a range of human tumors, including bladder, head and neck, lung, ovarian, and testicular cancers[69]. A previous study showed that CDDP could induce POI injury due to the excessive activation of dormant primordial follicles via the PTEN/AKT/ FOXO3a pathway[10-12]. Furthermore, we previously reported that CDDP-induced endoplasmic reticulum stress (ERS) could promote autophagy and apoptosis 
in granulosa cells (GCs), thus, resulting in excessive follicle loss and endocrine disorders[13]. Therefore, predicting the development of ovarian injury during CDDP chemotherapy remains a significant challenge.

The FK506-binding protein 4 (FKBP4, also known as FKBP52) protein is a member of the FK506-binding family of immunophilins, and serves as a co-chaperone for steroid hormone nuclear receptors to regulate endocrine function in target tissues[14-16]. The predominant role of FKBP4 is to regulate the steroid hormone receptor pathway, including receptor binding and transport, but is also associated with a various forms of cancer, and certain reproductive and neurological diseases[17, 18]. Deficiency of FKBP4 is known to compromise the fertilizing capacity of sperm[19] and promotes endometriosis[20]. Furthermore, FKBP4 deficiency conferred uterine progesterone resistance is specific to certain pregnancy stages, and is known to have a genetic background[21, 22].

MicroRNAs (miRNAs) are a class of endogenous noncoding RNAs (ncRNAs) that can mediate posttranslational silencing of the genes. Nowadays, microRNAs (miRNAs) are known to exhibit regulatory actions in the development of POI[23, 24]. However, the function of micro-483-5p (miR-483-5p) in ovarian was little known. Moreover, several studies have shown that miR-483-5p is associated with the progression of various cancers[25]. For example, the modulation of $m i R-483-5 p$ levels may increase CDDP sensitivity in tongue squamous cell carcinoma (TSCC) cells through a novel mitochondrial fission pathway[26]. miR-483-5p responds to the loss of chondrocyte matrix interaction by inhibiting the MAPK pathway[27]. Levels of miR-483-5p are known to be significantly reduced in the cumulus cells of patients with polycystic ovarian syndrome. This suggests that miR-483-5p might promote the proliferation of cumulus cells by activating the PI3K/AKT pathway, and that miR-483-5p may play an important role in reducing insulin resistance[28-30].

In this study, we investigated the relationship between FKBP4 and miR-483-5p, a possible regulator of FKBP4, and identified the mechanisms underlying their association with CDDP-induced POI. We found that FKBP4 was down-regulated in the CDDP-induced model, while miR-483-5p was up-regulated. The aim of this study was to elucidate the relationship between FKBP4 and miR-483-5p in the development of $\mathrm{POI}$ induced by CDDP.

\section{Methods}

\section{Reagents}

TRIzol LS reagent (Cat: 10296028) and lipofectamine 3000 (Cat: 130000) were purchased from Invitrogen (Waltham, MA, USA). The Realstar power SYBR kit (a311) was acquired from Genstar Biosolutions. miR-483-5p mimics, miR-483-5p hairpin-it real-time PCR kit, and U6 snRNA real-time PCR normalization kit (e01005) were purchased from Gene Pharma (Shanghai, P.R. China). qPCR primers were purchased from Sangong Biotech (Shanghai, P.R. China). The FKBP4 overexpression plasmid was obtained from Genechem (Shanghai, P.R. China). Horseradish peroxidase-conjugated goat anti-mouse IGG (115-035), anti-rabbit IGG (115-035), Alexa Fluor ${ }^{\circledR} 488$-conjugated donkey anti-rabbit IGG $(\mathrm{H}+\mathrm{L})(711$ - 
545), and Alexa Fluor ${ }^{\circledR}$ 594-conjugated donkey anti-rabbit IGG $(\mathrm{H}+\mathrm{L})$ (711-585) were obtained from Jackson Immunoresearch (West Grove, PA, USA). Dual-luciferase reporter assay system (e1910), and Deadend $^{\mathrm{TM}}$ fluorometric tunel system (g3250), were purchased from Promega (Madison, WI, USA). Xhol and Nothestriction enzymes were purchased from Thermo Fisher Scientific (Waltham, MA, USA). We also purchase mouse AMH (anti-Mullerian hormone) ELISA kit (e-el-m0113c), mouse FSH (follicle stimulating hormone) ELISA kit (e-el-m0511c), and human/monkey/mouse $E_{2}$ (estradiol) ELISA kit (e-el-0150c) from Elabscience (Houston, Texas, USA). Maintenance medium, consisting of Dulbecco's Modified Eagle Medium (DMEM) and fetal bovine serum, was purchased from Gibco (Waltham, MA, USA). All other reagents were purchased from Sigma-Aldrich (Darmstadt, Germany).

\section{Human serum collection}

Six unrelated Han Chinese POI women were recruited in this experiment, of whom suffered secondary amenorrhea and with elevated levels of FSH $>25 \mathrm{IU} / \mathrm{L}$ on two occasions at least one month apart, prior to 40 years-of-age. Patients were excluded with known chromosomal abnormalities, previous ovarian surgery, autoimmune disorders, somatic anomalies (particularly any reported as associated with syndromic POI) or radiotherapy and chemotherapy. Serum samples were obtained from normal and POI patients attending Shenzhen Second People's Hospital, Reproductive Medicine Centre (Shenzhen, China). All research involving human participants was approved by the Ethics Committees of Shenzhen Second People's Hospital (45575561-0, Shenzhen, China) and informed consent was obtained from all participants.

\section{The model of CDDP induced POI}

Wild-type female C57BL/6J mice (4-week-old) were purchased from the Southern Medical University Animal Center (Guangzhou, China). All mice were housed in a constant light-to-dark ratio of $12: 12 \mathrm{~h}$ under specific pathogen-free conditions in a university animal facility. Experimental POI was induced in 5-weekold females. The POI group received intraperitoneal injections of CDDP $(2.5 \mathrm{mg} / \mathrm{kg} / \mathrm{d}$ in saline $)$ for 7 consecutive days, while the control group was injected with saline[10]. The diagrammatic representation of CDDP administration is shown in Figure $S 1$.

\section{Trypsin digestion and iTRAQ labeling}

iTRAQ labeling was performed according to the manufacturer's protocol (Applied Biosystems, Sciex)[13]. Briefly, $200 \mu \mathrm{g}$ of each protein sample was reduced with TCEP Reducing Reagent at $60^{\circ} \mathrm{C}$ for $1 \mathrm{~h}$, and alkylated with MMTS Cysteine-Blocking Reagent at room temperature for $30 \mathrm{~min}$. Then, proteins were digested with trypsin (Promega, USA) at $37^{\circ} \mathrm{C}$ at a ratio of 1:50 (enzyme-to-substrate) overnight. Each sample was labeled separately with two of the eight available tags (control: 114 and 116 tags; cisplatin: 117 and 119 tags). All labeled peptides were pooled together.

\section{Generation of $m i R-483-5 p$ transgenic mice}


miR-483-5p-floxed mice were obtained from Cyagen Biosciences (Guangzhou, China). We also purchased Gdf9-Cre mice from the Jackson Laboratory (stock no.011062). The Gdf9-Cre mice were mated with miR483-5p-floxed mice to create female miR-483-5p transgenic mice (miR-483-5p TG), which exhibited oocyte-specific overexpression of miR-483-5p (Gdf9-Cre ${ }^{+}$, miR-483-5p TG). Females from the same litter that possessed miR-483-5p-LoxP, but without Gdf9-Cre (Gdf9-Cre-' miR-483-5p-LoxP), were used as controls. DNA was isolated from tail biopsies and genotyped by PCR.

\section{Real-time quantitative PCR (qRT-PCR)}

Total RNA and miRNA were purified with RNAiso Plus reagent, and TRIzol LS reagent, respectively. Then, total RNA was reverse transcribed into cDNA with the RETRO-script Reverse Transcription Kit, and qRTPCR was performed, in triplicate reactions, on a StepOne Plus Real-Time PCR System (Applied Biosystems, Waltham, MA, USA), using the RealStar Power SYBR Kit. Next, miR-483-5p was reversed transcribed and used for quantitative RT-PCR with the Hairpin-it Real-Time PCR kit. Quantitative expression data were then acquired for the ratio of FKBP4 to Gapdh (FKBP4/ Gapdh); U6 and Gapdh genes were used as endogenous controls in order to normalize the data for differences in the amount of total miRNA and RNA. We also determined miR-483-5p-tissue (cell)/U6 and miR-483-5p-serum/miR-39-3p ratios. Data were acquired using the $\Delta \Delta \mathrm{Ct}$ method [31] and used to determine the fold change in expression between the POI and control groups. The primers used for qRT-PCR were as follows: has-miR483-5p: forward:5ל-AGAGCACAAGACGGGAGGAA-3c, reverse:5ל-TATGGTTGTTCACGACTCCTTCAC-3द, mmu-miR-483-5p: forward: 5ל-CCACCTAAGACGGGAGAAGA-3c, reverse:5לे-

TATGGTTGTTGTGCTCTCTGACTC-3द, U6 snRNA: forward: 5ל-CGCTTCGGCAGCACATATAC-3द, reverse: 5ל-TTCACGAATTTGCGTGTCATC-3c, Cel-miR-39-3p: forward: 5c-CGTCGATCACCGGGTGTAAA-3c,

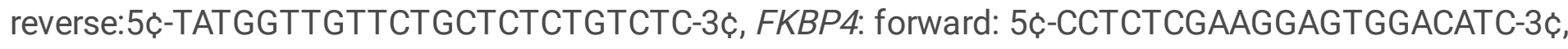
reverse: 5ל-TCCCCGATCATGGGTGTCT-3 $\dot{c}$, Gapdh: forward: 5ל-TGTGTCCGTCGTGGATCTGA -3c, reverse:

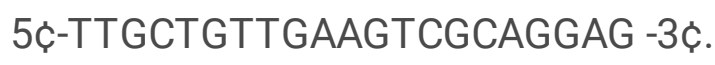

\section{Cultivation of primary GCs}

C57B6/J mice were injected with PMSG (5 IU, Sigma, St. Louis, MO) to increase GCs number, and hCG (5 IU, Sigma, St. Louis, MO) was injected after 48 hours later then kill the mouse after 14 hours. Ovaries were removed and the GCs were extracted by puncturing ovaries. Collected GCs were centrifuged by Trypsin Solution (SangonBiotech) $\left(37^{\circ} \mathrm{C}, 3 \mathrm{~min}\right)$, then add DMEM/F12 Medium with $10 \%$ FBS into the cell suspension. GCs were maintained at $37^{\circ} \mathrm{C}$ in a humidified atmosphere containing $5 \% \mathrm{CO}_{2}$. Cells that have the same growth were randomly divided into four groups: control group, miR-483-5p group, miR-483$5 p+F K B P 4+C D D P$ group and miR-483-5p+CDDP group.

\section{Cell line, DNA transfection, and miRNA interference}

The HeLa (human cervical cancer cells) cell line was cultured in maintenance medium consisting of DMEM supplemented with $10 \%$ fetal bovine serum (FBS) and the KGN (human granulosa cells) cell line and primary GCs was cultured in maintenance medium consisting of DMEM/F12 supplemented with 10\% 
FBS. The cells were maintained under standard cell culture conditions of $5 \% \mathrm{CO}_{2}$ and $95 \%$ humidity. The miR-483-5p mimics, and the FKBP4 overexpression plasmid, were transfected using Lipofectamine 3000 in accordance with the manufacturer's instructions.

\section{Western blotting}

After treatment, ovaries were homogenized and lysed for $10 \mathrm{~min}$ at $100^{\circ} \mathrm{C}$ in buffer $(62.5 \mathrm{mM} \mathrm{Tris}-\mathrm{HCl}[\mathrm{pH}$ 6.8], $10 \%$ glycerol, $2 \%$ SDS, $50 \mathrm{mM} \mathrm{DTT}$, and $0.01 \%$ bromophenol blue). Cell and tissue lysates were then separated by $8-12.5 \%$ SDS-PAGE, and electro-transferred to nitrocellulose membranes (GE Healthcare Life Sciences, Beijing, China).The membranes were then blocked in $5 \%$ non-fat dried milk for $1 \mathrm{~h}$ at room temperature, washed, and incubated with rabbit polyclonal anti-FKBP4 (1:1000, ABclonal, A5643) primary antibody, rabbit polyclonal anti-PARP (1:500, CST, 9542S), and mouse polyclonal $\beta$-Actin (MG3) mouse monoclonal antibody (1:3000, Ray antibody Biotech, RM2001) at $4^{\circ} \mathrm{C}$ overnight. The membranes were then washed, incubated with secondary antibodies for $1 \mathrm{~h}$ at room temperature, and immunoreactive proteins visualized using an Enhanced Chemiluminescence Kit (Bio-Rad). $\beta$-Actin served as an internal control. The quantitative analysis of protein expression was carried out with ImageJ software 1.8.0 ( $\mathrm{NIH}$, Bethesda, Maryland, USA).

\section{In situ hybridization}

The sequence of the probe (Exiqon) for $m m u-m i R-483-5 p$, containing the locked nucleic acid and digoxigenin-modified bases, was: /5DigN/CTCCCTTCTCTTCTCCCGTCTT/3Dig_N/. Signals were detected using anti-Digoxigenin-AP (Roche), NBT/BCIP was used as a chromogen, and images were acquired using an AXIO Scope A1 (Zeiss). Densitometry analysis of the in situ hybridization images were performed with ImageJ software 1.8.0.[32].

\section{Luciferase assay}

The FKBP4 mRNA 3ל untranslated region (3ל्CTR) (GenBank: NM_010219.4) was amplified from mouse CDNA, and the mutation binding region was amplified using mutant primers. PCR products, and the empty psiCHECK-2 vector, were then digested with $\mathrm{Xhol}$ and Not/ restriction enzymes and subsequently ligated by T4 ligase. The primer sequences were as follows: FKBP4 mRNA 3द्CUTR: forward: $5 \dot{c}-$ CCGCTCGAGGGGTGGAGACAGAAGCGTAG-3द, reverse: $5 \dot{c}$ -

ATTTGCGGCCGCTGACACCATCTAAAACTACCCCC-3ל, FKBP4 mutant binding region: forward: $5 \bar{\zeta}-$ CCGCTCGAGCTCGGGTGGGTGGAGACAGA-3द, reverse: $5 \dot{\zeta}$ -

ATTTGCGGCCGCAAGAAAAGTAGGGTTGAGAGG-3C. miR-483-5p mimics and/or the FKBP4 plasmid were then transfected with Lipofectamine 3000 in accordance with the manufacturer's instructions.

The FKBP4 mRNA 3द्CUTR expression or mutant binding region plasmids were co-transfected with miR483-5p, or negative control (NC) mimics into HeLa cells, respectively. Cells were then analyzed with dualluciferase reporter assay system, that was applied in accordance with the manufacturer's instructions. Luminescent signals were quantified using a luminometer (Glomax, Promega)[33]. 


\section{Tissue collection and morphological analysis}

Tissues were first fixed in $4 \%$ Paraformaldehyde solution for $24 \mathrm{~h}$ and then embedded in paraffin wax. Sections of $4 \mu \mathrm{m}$ thickness were then prepared for hematoxylin and eosin (H\&E) staining. At least five sections (taken $100 \mu \mathrm{M}$ apart) representative sections from each ovary each group were photographed for follicular assessment. A follicle was deemed to be present if the oocyte contained a germinal vesicle. Follicles were counted and classified according to their health status and development stage, as either a healthy follicle (primordial, primary, secondary, or antral follicle), or an atretic follicle, in accordance with classifications described previously[34].

\section{The terminal deoxynucleotidyl transferase mediated dUTP nick-end labeling (TUNEL) assay}

For TUNEL analysis, sections were first deparaffinized, and then rehydrated. Sections were then permeabilized with $20 \mu \mathrm{g} / \mathrm{mL}$ of proteinase $\mathrm{K}$, and incubated with TUNEL reagents in accordance with the manufacturer's guidelines. Next, the sections were counterstained with DAPI. TUNEL-positive signals were then quantified. Every 25th section in each ovary were analyzed, using the incubation with the enzyme as a positive control, or without the enzyme as a negative control. Images were acquired with a FluoView FV1000 confocal microscope (Olympus, Tokyo, Japan). The relative level of apoptosis was presented as the proportion of apoptotic cells in granulosa cells on each follicle.

\section{Immunofluorescence staining}

For immunofluorescence staining, sections were first deparaffinized and then rehydrated. Next, $4 \mu \mathrm{m}$ sections were incubated with rabbit polyclonal anti-connexin 43 primary antibody (1:100, Immunoway, YT1046), rabbit polyclonal anti-connexin 37 primary antibody (1:100, ABclonal, A2529), anti-ki67 primary antibody (1:300, ABclonal, 9129), or anti-FKBP4 (1:100, ABclonal, A5643) primary antibody. This was then followed by incubation with Alexa-Fluor-488- or Alexa-Fluor-594-labeled secondary antibody, as appropriate. Finally, the sections were counterstained with DAPI and immunofluorescent images were acquired using a FluoView FV1000 confocal microscope (Olympus, Tokyo, Japan).

\section{Enzyme-linked immunosorbent assays (ELISA)}

For ELISA, we first separated the supernatant from blood samples by centrifugation at $3000 \times \mathrm{g}$ for 10 min. Next, we used ELISA to determine the levels of $A M H, F S H$, or $E_{2}$; for this we used specific kits for each hormone in accordance with the manufacturer's instructions. The absorbance of the reaction mixture was determined with a spectrophotometer at $450 \mathrm{~nm}$. Hormone concentrations were determined from standards by means of a four-parameter logistic curve drawn on log-log graph paper.

\section{Statistical analysis}

Statistical analysis was calculated using GraphPad Prism version 8.0 (GraphPad Software, San Diego, CA). Data are given as mean \pm SEM. The Student's t-test was used to compare data between the two 
groups of samples. One-way analysis of variance (ANOVA), and the post hoc Tukey test, were used to analyze samples when $\geq 3$ groups were involved. $P<0.05$ was considered to be statistically significant.

\section{Results}

The ovaries of POI mice, induced by CDDP, exhibited reduced expression levels of FKBP4 mRNA and protein

To investigate the specific mechanisms underlying POI, we administered C57BL/ 6 mice with CDDP and then harvested their ovaries. Figure $S 1$ shows how the POI mouse model was generated. Proteomic screening, by isobaric tags for relative and absolute quantification analysis (iTRAQ) analysis, identified that the FKBP4 protein significantly down-regulated in the ovaries from the CDDP-induced group (fold change $=-0.6503$ ) compared to the control group treated with normal saline (Fig. 1a). Furthermore, the expression level of FKBP4 mRNA was significantly lower in the ovaries from the CDDP-induced POI model than the controls $(P<0.01$, Fig. $1 \mathrm{~b})$. We also found that the expression level of FKBP4 protein was significantly reduced in the CDDP-induced POI mouse model $(P<0.05$, Fig. $1 \mathrm{c}$ and $\mathrm{d})$. Therefore, these results suggest that the expression of FKBP4 is down-regulated in the ovaries of mice in which POI had been induced by CDDP.

\section{miR-483-5p is the likely upstream regulator for FKBP4 and is expressed at high levels in both the CDDP- POI mouse model, and patients with POI}

To identify the potential mechanism underlying the down-regulation of FKBP4 protein in CDDP-induced $\mathrm{POI}$, we used TargetScan software to identify a potential upstream regulator, miR-483-5p, which could specifically bind to the 3c्CTR 1771-1765 sequences of FKBP4 mRNA (Fig. 2a). Next, we used qRT-PCR to determine the expression levels of miR-483-5p in the ovaries, and serum, from the CDDP-induced mouse model. Analysis showed that the relative expression levels of $m i R-483-5 p$ were significantly higher in the ovaries $(P<0.01$, Fig. 2 b), and serum $(P<0.001$, Fig. 2 c), of the CDDP-induced mouse model. In situ hybridization experiments further found that miR-483-5p was highly expressed in oocytes and GCs from the CDDP-induced model than that in the saline-treated group $(P<0.001$, Fig. $2 \mathrm{~d}$ and e). Moreover, we collected serum from patients with POI and determined the miR-483-5p content of these samples using qRT-PCR. Compared with the group of healthy patients, the relative expression of $m i R-483-5 p$ was significantly higher in patients with $\mathrm{POI}(P<0.001$, Fig. 2f); these findings were consistent with those generated by the CDDP-POI mouse model. Therefore, these data indicate that the down-regulation of FKBP4 may be caused by the up-regulation of miR-483-5p during the pathogenesis of $\mathrm{POI}$.

\section{FKBP4 is the functional target of miR-483-5p and FKBP4 could alleviate GCs apoptosis by CDDP in vitro}

To determine whether miR-483-5p acts as the regulator for FKBP4 protein, we carried out transfection experiments with HeLa cells, this effect was verified by qRT-PCR $(P<0.001$, Fig. 3a). Dual-luciferase assays demonstrated that nucleotides 1765-1771 of the 3CUTR of the human FKBP4 protein were complementary to the miR-483-5p seed sequences. This region was shown to inhibit the luciferase 
activation of a reporter plasmid containing the FKBP43CUTR mimics. However, there was no obvious effect when the reporter plasmid contained the mutant 3 CLTR, as this was unable to combine with miR483-5p ( $P<0.01$, Fig. 3b). Furthermore, levels of FKBP4 protein were significantly reduced in HeLa cells that had been transfected with miR-483-5p mimics ( $P<0.01$, Fig. $3 \mathrm{c}$ and d).

In order to investigate the relationship between miR-483-5p and FKBP4 with regards to the pathogenesis of CDDP-induced POI, we over-expressed miR-483-5p, or FKBP4 alone, or miR-483-5p and FKBP4 simultaneously, in KGN cells and primary GCs. We found that the levels of the FKBP4 protein were downregulated in KGN cells and primary GCs following the overexpression of miR-483-5p, with or without CDDP treatment ( $P<0.01$, Fig. 4 a and b, $P<0.05, P<0.05$, Fig. $4 \mathrm{~d}$ and e). However, the expression levels of PARP were significantly up-regulated in KGN cells and primary GCs following the overexpression of miR-483-5p, with or without CDDP treatment ( $P<0.01$, Fig. 4 a and c, $P<0.05$, Fig. $4 \mathrm{~d}$ and f). Furthermore, the overexpression of FKBP4 in KGN cells and primary GCs were shown to reverse the up-regulation of PARP expression induced by the overexpression of miR-483-5p with $\operatorname{CDDP}(P<0.01$, Fig. 4a and $\mathrm{c})$. These findings indicated that the overexpression of FKBP4 could alleviate apoptosis in KGN cells and primary GCs caused by the overexpression of miR-483-5p induced by CDDP.

\section{The overexpression of miR-483-5p in oocytes aggravated ovarian injury and disrupted the levels of sex hormones in the POI mouse model induced by CDDP}

Next, we generated transgenic mice that overexpressed $m i R-483-5 p$ in an oocyte-specific manner to investigate the relationship between miR-483-5p and FKBP4 in vivo (Fig. 5a and b). Our analysis demonstrated that the expression levels of miR-483-5p were significantly higher in oocytes and GCs from miR-483-5p TG mice, as determined by in situ hybridization and semi-quantification analysis $(P<0.01$, Fig. $5 c$ and d). Interestingly, the expression levels of FKBP4 were significantly reduced in both the oocytes and of miR-483-5p TG mice ( $P<0.05$, Fig. $5 e$ and $\mathrm{f})$. Furthermore, the levels of FKBP4 protein were further reduced in miR-483-5p TG mice when treated with $\operatorname{CDDP}(P<0.01$, Fig. 5e and f).

Although there was a reduction in the weight of the ovaries in miR-483-5p TG mice, there was no significant difference in terms of ovarian weight loss when compared with the control group (Fig. $6 \mathrm{a}$ and b). It is possible that the ovarian weight loss could have been caused by the CDDP treatment. However, CDDP had a more significant effect on the weight loss of ovaries in the miR-483-5p TG mice $(P<0.05$, Fig. $6 a$ and b). Next, H\&E staining was used to detect changes in ovarian morphology and follicle count (Fig. 6c). Histological analysis showed that the number of normal follicles, particularly primary, secondary and antral follicles, fell significantly in miR-483-5p TG mice following CDDP treatment $(P<$ 0.05 , Fig. $6 \mathrm{~d})$, at the same time, there was a significant increase in the number of atretic follicles in this group of mice $(P<0.01$, Fig. 6e). In addition, the levels of AMH and estrogen were significantly reduced in miR-483-5p TG mice after CDDP treatment $(P<0.05, P<0.01$, Fig. $6 \mathrm{f}$ and $\mathrm{g})$, while the levels of FSH were significantly increased $(P<0.05, P<0.01$, Fig. $6 \mathrm{~h}$ ). Immunofluorescence analysis further showed that there was a significant tendency for increased levels of apoptosis, and down-regulation in proliferation ability, in granulosa cells from miR-483-5p TG mice following CDDP treatment $(P<0.05, P<0.01$, Fig. 61 , j 
and $\mathrm{m}, \mathrm{n}$ ). Our analysis also showed that both levels of Connexin 37 (Cx37) and Connexin 43 (Cx43) were reduced in this group, indicating that gap junctions between oocyte-granulosa cells and granulosagranulosa cells were broken $(P<0.05, P<0.01$, Fig. $6 \mathrm{k}, \mathrm{I}$ and $\mathrm{o}, \mathrm{p})$. These results suggested that the miR483-5p TG mice may be more sensitive to CDDP, particularly with regards to ovarian damage.

\section{Discussion}

$\mathrm{POI}$ is one of the causes of infertility, and the great challenge is that it is difficult to cure or reverse. In addition, patients with $\mathrm{POI}$ can often exhibit serious mental and physical problems[35, 36]. One of the major causes is that chemotherapy drugs were applied to the treatment of childhood cancer. In recent decades, childhood cancer 5-year survival rate have markedly increased, also, adulthood infertility rate has remarkably increase[37]. A study showed that imatinib protects mouse oocytes from chemotherapyinduced death by inhibiting the pathway of c-Abl-TAp63[38]. Furthermore, it was confirmed that imatinib could provide selective protection against CDDP induced ovarian injury[39]. However, there is an urgent need to identify preventive diagnostic markers and effective treatments for $\mathrm{POI}$ patients. There is a growing body of evidence to suggest that microRNAs (miRNAs) are important regulators in the pathogenesis of POI[23]. Hence, the purpose of this study was to establish a CDDP-induced POI animal model, and to elucidate the functional pathway of miRNA in the development of POI.

In order to explore the mechanism underlying the development of POI, we established a mouse model of POI by administering CDDP. Our analysis identified a protein, FKBP4, that was significantly downregulated in the ovaries of the CDDP-induced model, as determined by proteomic screening/iTRAQ analysis. As a member of the family of FK506-binding proteins (FKBPs), FKBP4 is able to associate with receptor-HSP90 complexes, and has been shown to act as an important positive regulator for androgen, glucocorticoid, and progesterone receptor signaling pathways[15]. However, FKBP4 is also associated with various types of cancer, along with certain reproductive and neurological diseases[17, 18]. Our analysis demonstrated reduced expression levels of both FKBP4 mRNA and protein in the ovaries of the CDDP-induced POI model. Recent studies have also demonstrated that both male and female FKBP4deficient mice exhibit characteristics of androgen, glucocorticoid, and/or progesterone insensitivity[40]. Therefore, the down-regulation of FKBP4 in the ovaries may play a role in the pathological process of CDDP-induced POI.

To further clarify why FKBP4 was down-regulated in CDDP-induced POI, we used TargetScan software and identified $m i R-483-5 p$ as an upstream endogenous regulator of FKBP4. Firstly, we found that miR483-5p was CDDP treatment induced high expression levels of miR-483-5p in the ovaries, as determined by qRT-PCR. Interestingly, levels of miR-483-5p were also up-regulated in POI patients in comparison with normal fertile controls. According to the existing literature, the up-regulation of miR-483-5p is significantly associated with poorer disease-specific survival in adrenocortical carcinomas[41]. miR-483-5p has also been shown to be essential for the initiation and development of osteoarthritis (OA) by stimulating chondrocyte hypertrophy and angiogenesis[42]. In this study, our findings revealed that FKBP4 is a confirmed target for miR-483-5p in HeLa cells. We also found that the overexpression of FKBP4 in KGN 
cells could alleviate the apoptosis caused by CDDP and the overexpression of miR-483-5p. Furthermore, miR-483-5p could specifically bind to the 3C्UTR 1771-1765 sequences of FKBP4 mRNA. Therefore, our results indicate that $m i R-483-5 p$ controls the expression of FKBP4.

In order to investigate the function of miR-483-5p in the development of POI, we generated transgenic mice that over-expressed miR-483-5p in an oocyte-specific manner. However, no obvious pathological characteristics of $\mathrm{POI}$ were evident in this model when compared with the control. This indicated that the oocyte-specific overexpression of miR-483-5p alone could not induce the pathological characteristics of POI. It has previously been reported that mice over-expressing miR-483-5p did not show any abnormal structural changes in terms of liver morphology[43]. Next, miR-483-5p TG mice were treated with CDDP. We found that the severity of experimental POI induced by CDDP alone could be significantly enhanced by the overexpression of miR-483-5p. Rather, the overexpression of miR-483-5p in oocytes could enhance ovarian injury and disrupt sex hormone levels in the POI mouse model induced by CDDP. Our data also indicate that the oocyte-specific overexpression of miR-483-5p alone could not induce POI. However, when combined with CDDP treatment, the oocyte-specific overexpression of miR-483-5p could enhance the severity of POI than CDDP treatment alone. These data indicated that the miR-483-5p TG mice may be more sensitive to CDDP, particularly with regards to ovarian damage.

\section{Conclusions}

In summary, our findings revealed a novel $m i R-483-5 p / F K B P 4$ pathway that plays a role in the development of POI induced by CDDP. We also showed that the down-regulation of FKBP4, and the upregulation of miR-483-5p, may represent a meaningful event in the occurrence and development of CDDPinduced POI. Therefore, miR-483-5p TG mice may be more sensitive to CDDP, particularly with regards to ovarian damage. Therefore, detecting serum miR-483-5p represents an approach that could predict the onset of $\mathrm{POI}$ and its progression.

\section{Declarations}

\section{Acknowledgements}

We are grateful to Professor Hongyan Yang and Professor Yiming Mu for kindly providing the granulosa cell lines. We would like to thank the technicians in the Reproductive Medicine Centre of First Hospital Affiliated Shenzhen University for their help in sample collections, tissue processing, and we also thank the technical assistance of department of Cell Biology, Southern Medical University.

\section{Authors' contributions}

LPW and HHZ designed the experiments. $\mathrm{HHZ}$ and WQG conducted the experiments and carried out the statistical analysis with help from HGOY, WBP, HBZ and LS. HHZ and RYD wrote the manuscript. All of the authors read and approved the final manuscript. 


\section{Funding}

This research was supported by the National Natural Science Foundation of China (grant numbers: 81571389), Guangdong Basic and Applied Basic Research Foundation (grant number: 2019A1515011693).

\section{Availability of data and materials}

The datasets used and/or analyzed during the current study are available from the corresponding author on reasonable request.

\section{Ethics approval and consent to participate}

All animal experiments were approved by the Southern Medical University Committee on the Use and Care of Animals and were performed in accordance with the Committee's guidelines and regulations. All of the POI patients were approved by the Medical Ethical Review Committee of Shenzhen Second People's Hospital and informed consent was obtained from all participants.

\section{Consent for publication}

Not applicable.

\section{Competing interests}

None of the authors have any conflicts of interest to declare.

\section{Abbreviations}

premature ovarian insufficiency POI

follicle-stimulating hormone FSH

cis-diaminedichloroplatinum CDDP

endoplasmic reticulum stress ERS

granulosa cells GCs

FK 506-binding protein 4 FKBP4

tongue squamous cell carcinoma TSCC

Dulbecco's Modified Eagle Medium DMEM

isobaric tags for relative and absolute quantification analysis ITRAQ 
real-time quantitative PCR qRT-PCR

fetal bovine serum FBS

mRNA 3ç untranslated region 3द्CUTR

terminal deoxynucleotidyl transferase mediated dUTP nick-end labeling TUNEL

Enzyme-linked immunosorbent assays ELISA.

Estrogen $E_{2}$

Connexin $37 \mathrm{Cx} 37$

Connexin $43 \mathrm{Cx} 43$

FK506-binding proteins FKBPs

osteoarthritis $\mathrm{OA}$

\section{References}

1. European Society for Human R, Embryology Guideline Group on POI, Webber L, Davies M, Anderson R, Bartlett J, Braat D, Cartwright B, Cifkova R, de Muinck Keizer-Schrama S, et al: ESHRE Guideline: management of women with premature ovarian insufficiency. Hum Reprod 2016, 31:926-937.

2. Orlandini C, Regini C, Vellucci FL, Petraglia F, Luisi S: Genes involved in the pathogenesis of premature ovarian insufficiency. Minerva Ginecol 2015, 67:421-430.

3. Maclaran K, Panay N: Current concepts in premature ovarian insufficiency. Womens Health (Lond) 2015, 11:169-182.

4. Maciejewska-Jeske M, Szeliga A, Meczekalski B: Consequences of premature ovarian insufficiency on women's sexual health. Prz Menopauzalny 2018, 17:127-130.

5. Meirow D, Nugent $D$ : The effects of radiotherapy and chemotherapy on female reproduction. Hum Reprod Update 2001, 7:535-543.

6. Farooq MA, Aquib M, Farooq A, Haleem Khan D, Joelle Maviah MB, Sied Filli M, Kesse S, BoakyeYiadom KO, Mavlyanova R, Parveen A, Wang B: Recent progress in nanotechnology-based novel drug delivery systems in designing of cisplatin for cancer therapy: an overview. Artif Cells Nanomed Biotechnol 2019, 47:1674-1692.

7. Roberts CM, Tran MA, Pitruzzello MC, Wen W, Loeza J, Dellinger TH, Mor G, Glackin CA: TWIST1 drives cisplatin resistance and cell survival in an ovarian cancer model, via upregulation of GAS6, L1CAM, and Akt signalling. Sci Rep 2016, 6:37652.

8. Dasari S, Tchounwou PB: Cisplatin in cancer therapy: molecular mechanisms of action. Eur $J$ Pharmacol 2014, 740:364-378. 
9. Wang C, Gu M, Wang S, Ma D: Comparative study on three chemotherapeutic regimens for the treatment of advanced epithelial ovarian cancer. J Tongji Med Univ 2000, 20:343-344.

10. Chang EM, Lim E, Yoon S, Jeong K, Bae S, Lee DR, Yoon TK, Choi Y, Lee WS: Cisplatin Induces Overactivation of the Dormant Primordial Follicle through PTEN/AKT/FOXO3a Pathway which Leads to Loss of Ovarian Reserve in Mice. PLoS One 2015, 10:e0144245.

11. Jang H, Na Y, Hong K, Lee S, Moon S, Cho M, Park M, Lee OH, Chang EM, Lee DR, et al: Synergistic effect of melatonin and ghrelin in preventing cisplatin-induced ovarian damage via regulation of FOX03a phosphorylation and binding to the p27(Kip1) promoter in primordial follicles. $J$ Pineal Res 2017, 63.

12. Meirow D, Schiff E: Appraisal of chemotherapy effects on reproductive outcome according to animal studies and clinical data. J Natl Cancer Inst Monogr 2005:21-25.

13. Wu Y, Ma C, Zhao H, Zhou Y, Chen Z, Wang L: Alleviation of endoplasmic reticulum stress protects against cisplatin-induced ovarian damage. Reprod Biol Endocrinol 2018, 16:85.

14. Cox MB, Riggs DL, Hessling M, Schumacher F, Buchner J, Smith DF: FK506-binding protein 52 phosphorylation: a potential mechanism for regulating steroid hormone receptor activity. $\mathrm{Mol}$ Endocrinol 2007, 21:2956-2967.

15. Li P, Ding Y, Wu B, Shu C, Shen B, Rao Z: Structure of the N-terminal domain of human FKBP52. Acta Crystallogr D Biol Crystallogr 2003, 59:16-22.

16. Tranguch S, Cheung-Flynn J, Daikoku T, Prapapanich V, Cox MB, Xie H, Wang H, Das SK, Smith DF, Dey SK: Cochaperone immunophilin FKBP52 is critical to uterine receptivity for embryo implantation. Proc Natl Acad Sci U S A 2005, 102:14326-14331.

17. Hausch F: FKBPs and their role in neuronal signaling. Biochim Biophys Acta 2015, 1850:2035-2040.

18. Storer CL, Dickey CA, Galigniana MD, Rein T, Cox MB: FKBP51 and FKBP52 in signaling and disease. Trends Endocrinol Metab 2011, 22:481-490.

19. Hong J, Kim ST, Tranguch S, Smith DF, Dey SK: Deficiency of co-chaperone immunophilin FKBP52 compromises sperm fertilizing capacity. Reproduction 2007, 133:395-403.

20. Hirota Y, Tranguch S, Daikoku T, Hasegawa A, Osuga Y, Taketani Y, Dey SK: Deficiency of immunophilin FKBP52 promotes endometriosis. Am J Pathol 2008, 173:1747-1757.

21. Demetriou C, Chanudet E, Gosgene, Joseph A, Topf M, Thomas AC, Bitner-Glindzicz M, Regan L, Stanier P, Moore GE: Exome sequencing identifies variants in FKBP4 that are associated with recurrent fetal loss in humans. Hum Mol Genet 2019, 28:3466-3474.

22. Tranguch S, Wang H, Daikoku T, Xie H, Smith DF, Dey SK: FKBP52 deficiency-conferred uterine progesterone resistance is genetic background and pregnancy stage specific. J Clin Invest 2007, 117:1824-1834.

23. Guo Y, Sun J, Lai D: Role of microRNAs in premature ovarian insufficiency. Reprod Biol Endocrinol 2017, 15:38.

24. Li Y, Fang Y, Liu Y, Yang X: MicroRNAs in ovarian function and disorders. J Ovarian Res 2015, 8:51. 
25. Pepe F, Visone R, Veronese A: The Glucose-Regulated MiR-483-3p Influences Key Signaling Pathways in Cancer. Cancers (Basel) 2018, 10.

26. Fan S, Chen WX, Lv XB, Tang QL, Sun LJ, Liu BD, Zhong JL, Lin ZY, Wang YY, Li QX, et al: miR-483-5p determines mitochondrial fission and cisplatin sensitivity in tongue squamous cell carcinoma by targeting FIS1. Cancer Lett 2015, 362:183-191.

27. Yang M, Zhang L, Gibson GJ: Chondrocyte miRNAs 221 and 483-5p respond to loss of matrix interaction by modulating proliferation and matrix synthesis. Connect Tissue Res 2015, 56:236-243.

28. Gallo W, Esguerra JLS, Eliasson L, Melander O: miR-483-5p associates with obesity and insulin resistance and independently associates with new onset diabetes mellitus and cardiovascular disease. PLoS One 2018, 13:e0206974.

29. Shi L, Liu S, Zhao W, Shi J: miR-483-5p and miR-486-5p are down-regulated in cumulus cells of metaphase II oocytes from women with polycystic ovary syndrome. Reprod Biomed Online 2015, 31:565-572.

30. Song S, Tan Y: Expression of FKBP52 in the ovaries of PCOS rats. Int J Mol Med 2019, 43:868-878.

31. Dubey R, Malhotra SS, Gupta SK: Forskolin-mediated BeWo cell fusion involves down-regulation of miR-92a-1-5p that targets dysferlin and protein kinase cAMP-activated catalytic subunit alpha. Am J Reprod Immuno/ 2018, 79:e12834.

32. Kivihall A, Aab A, Soja J, Sladek K, Sanak M, Altraja A, Jakiela B, Bochenek G, Rebane A: Reduced expression of miR-146a in human bronchial epithelial cells alters neutrophil migration. Clin Trans/ Allergy 2019, 9:62.

33. Song Q, Xu Y, Yang C, Chen Z, Jia C, Chen J, Zhang Y, Lai P, Fan X, Zhou X, et al: miR-483-5p promotes invasion and metastasis of lung adenocarcinoma by targeting RhoGDI1 and ALCAM. Cancer Res 2014, 74:3031-3042.

34. Chen ZG, Luo LL, Xu JJ, Zhuang XL, Kong XX, Fu YC: Effects of plant polyphenols on ovarian follicular reserve in aging rats. Biochem Cell Biol 2010, 88:737-745.

35. Machura P, Grymowicz M, Rudnicka E, Pieta W, Calik-Ksepka A, Skorska J, Smolarczyk R: Premature ovarian insufficiency - hormone replacement therapy and management of long-term consequences. Prz Menopauzalny 2018, 17:135-138.

36. Wu X, Cai H, Kallianpur A, Li H, Yang G, Gao J, Xiang YB, Ji BT, Yu T, Zheng W, Shu XO: Impact of premature ovarian failure on mortality and morbidity among Chinese women. PLoS One 2014, 9:e89597.

37. Allen CM, Lopes F, Mitchell RT, Spears N: Comparative gonadotoxicity of the chemotherapy drugs cisplatin and carboplatin on prepubertal mouse gonads. Mol Hum Reprod 2020, 26:129-140.

38. Gonfloni S, Di Tella L, Caldarola S, Cannata SM, Klinger FG, Di Bartolomeo C, Mattei M, Candi E, De Felici M, Melino G, Cesareni G: Inhibition of the c-Abl-TAp63 pathway protects mouse oocytes from chemotherapy-induced death. Nat Med 2009, 15:1179-1185.

39. Morgan S, Lopes F, Gourley C, Anderson RA, Spears N: Cisplatin and doxorubicin induce distinct mechanisms of ovarian follicle loss; imatinib provides selective protection only against cisplatin. 
PLoS One 2013, 8:e70117.

40. Sivils JC, Storer CL, Galigniana MD, Cox MB: Regulation of steroid hormone receptor function by the 52-kDa FK506-binding protein (FKBP52). Curr Opin Pharmacol 2011, 11:314-319.

41. Soon PS, Tacon LJ, Gill AJ, Bambach CP, Sywak MS, Campbell PR, Yeh MW, Wong SG, Clifton-Bligh RJ, Robinson BG, Sidhu SB: miR-195 and miR-483-5p Identified as Predictors of Poor Prognosis in Adrenocortical Cancer. Clin Cancer Res 2009, 15:7684-7692.

42. Wang H, Zhang H, Sun Q, Wang Y, Yang J, Yang J, Zhang T, Luo S, Wang L, Jiang Y, et al: Intraarticular Delivery of Antago-miR-483-5p Inhibits Osteoarthritis by Modulating Matrilin 3 and Tissue Inhibitor of Metalloproteinase 2. Mol Ther 2017, 25:715-727.

43. Zhang Y, Wu D, Zhang X, Ma N, Liu Y: MicroRNA Sponge Knockdowns miR-483-5p and Upregulates Serum ALT/AST in Transgenic Mice. Biochemistry (Mosc) 2018, 83:54-59.

\section{Figures}

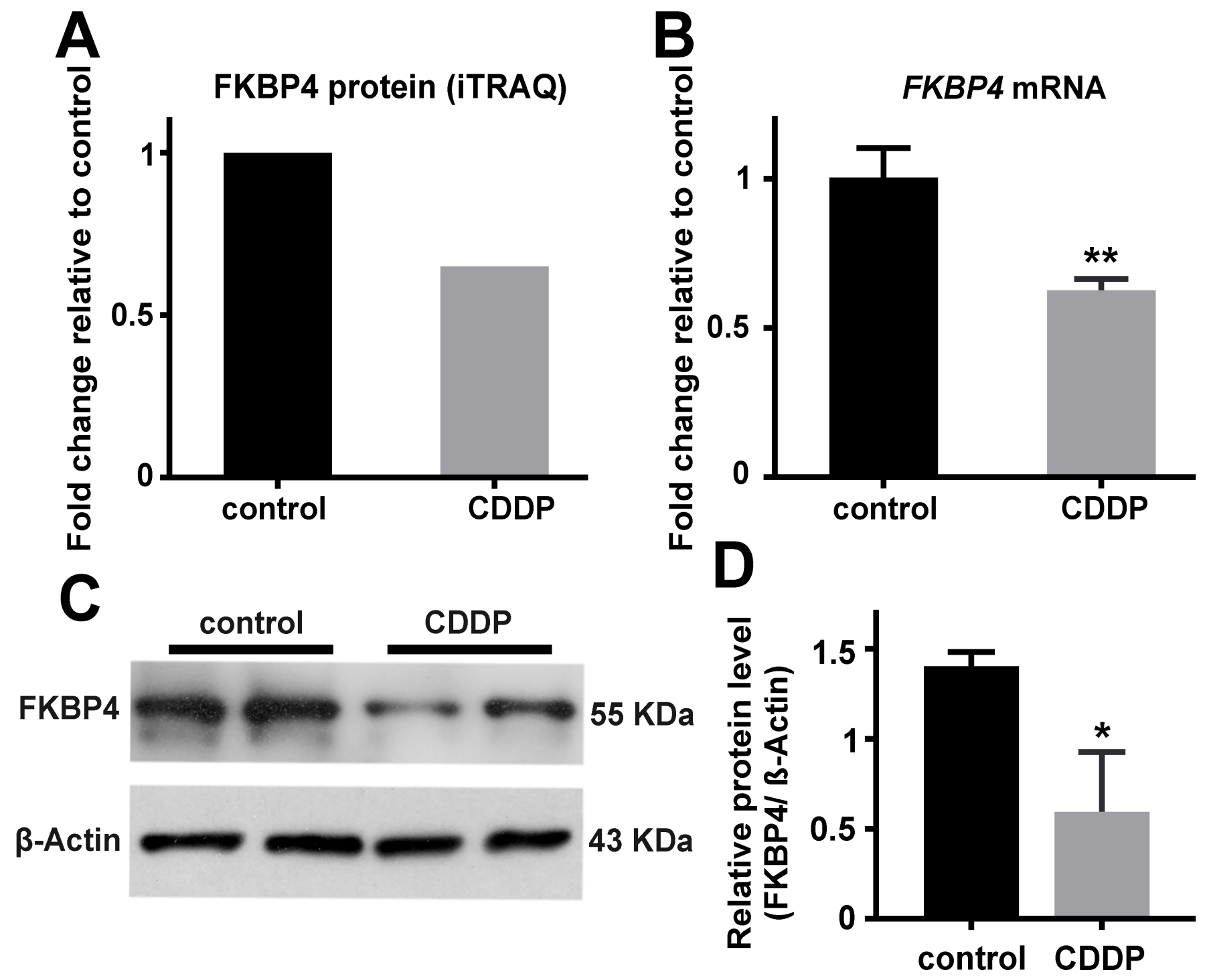

Figure 1 
The expression of FKBP4 was down-regulated in a CDDP-induced model of POI. a Reduced levels of FKBP4 were identified in the ovaries of the CDDP-induced POI model using iTRAQ analysis. b FKBP4 mRNA was detected in the ovaries of the CDDP-induced POI model using qRT-PCR. mRNA levels were normalized against Gapdh. $c$ The expression of FKBP4 protein was then determined in the ovaries of the CDDP-induced POI model using western blotting. $d$ Quantification analysis was normalized to $\beta$-Actin. All data are presented as mean \pm SEM. $n=5$ for each group. ${ }^{*} P<0.05,{ }^{*} P<0.01$, compared with controls.

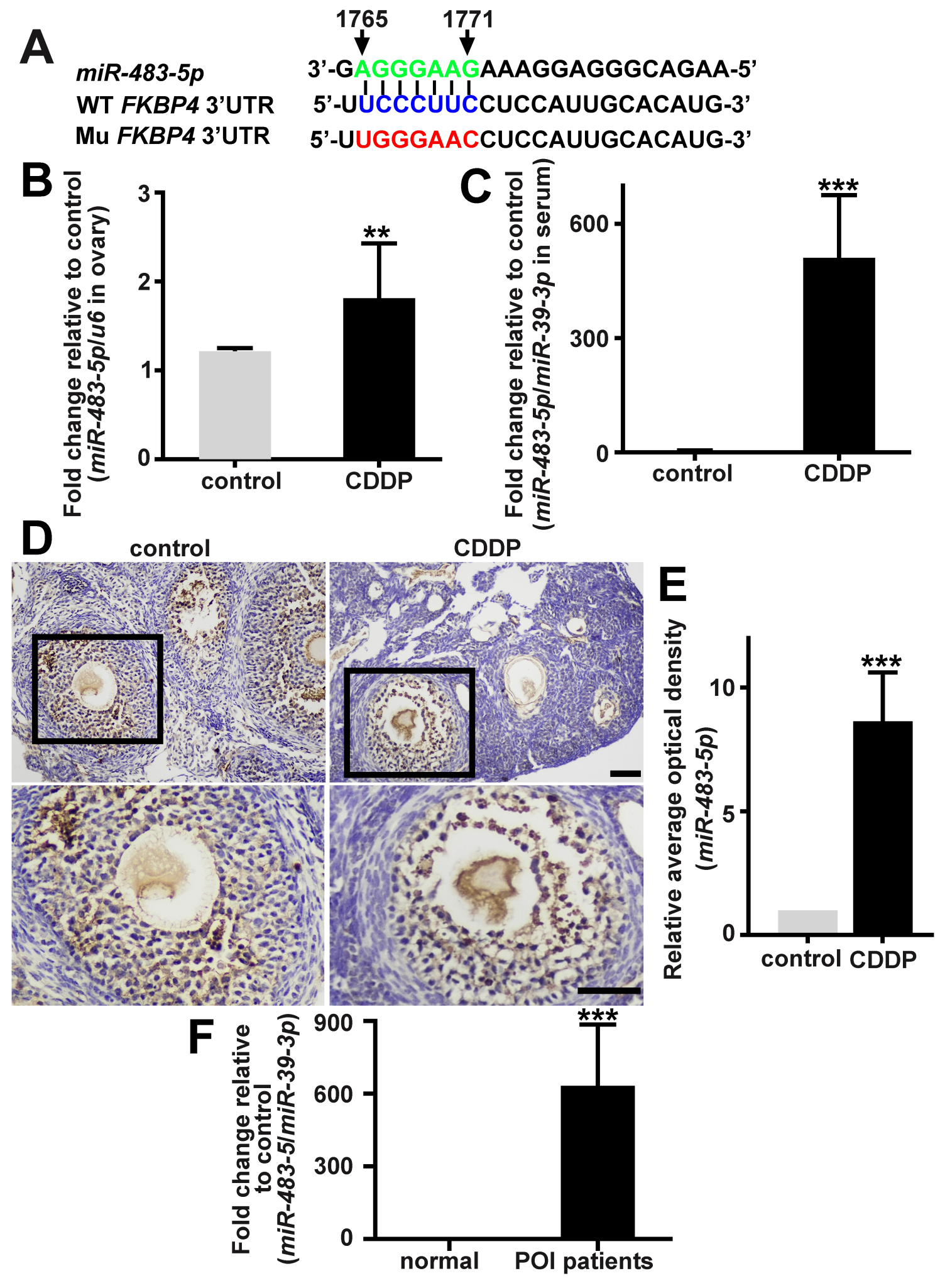

Figure 2 
miR-483-5p was highly expressed in the CDDP-POI model and the serum from POI patients. a miR-483-5p was predicted to be the deregulated microRNA responsible for FKBP4 and a schematic representation of the miR-483-5p in the 3'UTR from human FKBP4. Green represents the sequences from 1765 to 1771 of miR-483-5p, blue represents wild type sequences in the 3'UTR from human FKBP4; red represents mutant sequences in the 3'UTR from human FKBP4. A schematic representation of miR-483-5p in the 3 'UTR of FKBP4. The expression of miR-483-5p was determined by qRT-PCR in the $b$ ovaries and $c$ serum from a POI model induced by CDDP. miRNA levels were normalized against U6 and miR-39-3p. The location and expression levels of miR-483-5p were investigated in the ovaries of the POI model induced by CDDP using $d$ in situ hybridization and by e semi-quantification analysis. Images at the bottom represent enlarged views of the inserted frame. $f$ The expression of miR-483-5p was determined in the serum from POI patients using qRT-PCR. mRNA levels were normalized against miR-39-3p. All data are presented as mean \pm SEM. $n=5$ for each group. ${ }^{*} \mathrm{P}<0.01, * * * \mathrm{P}<0.001$, compared with controls.

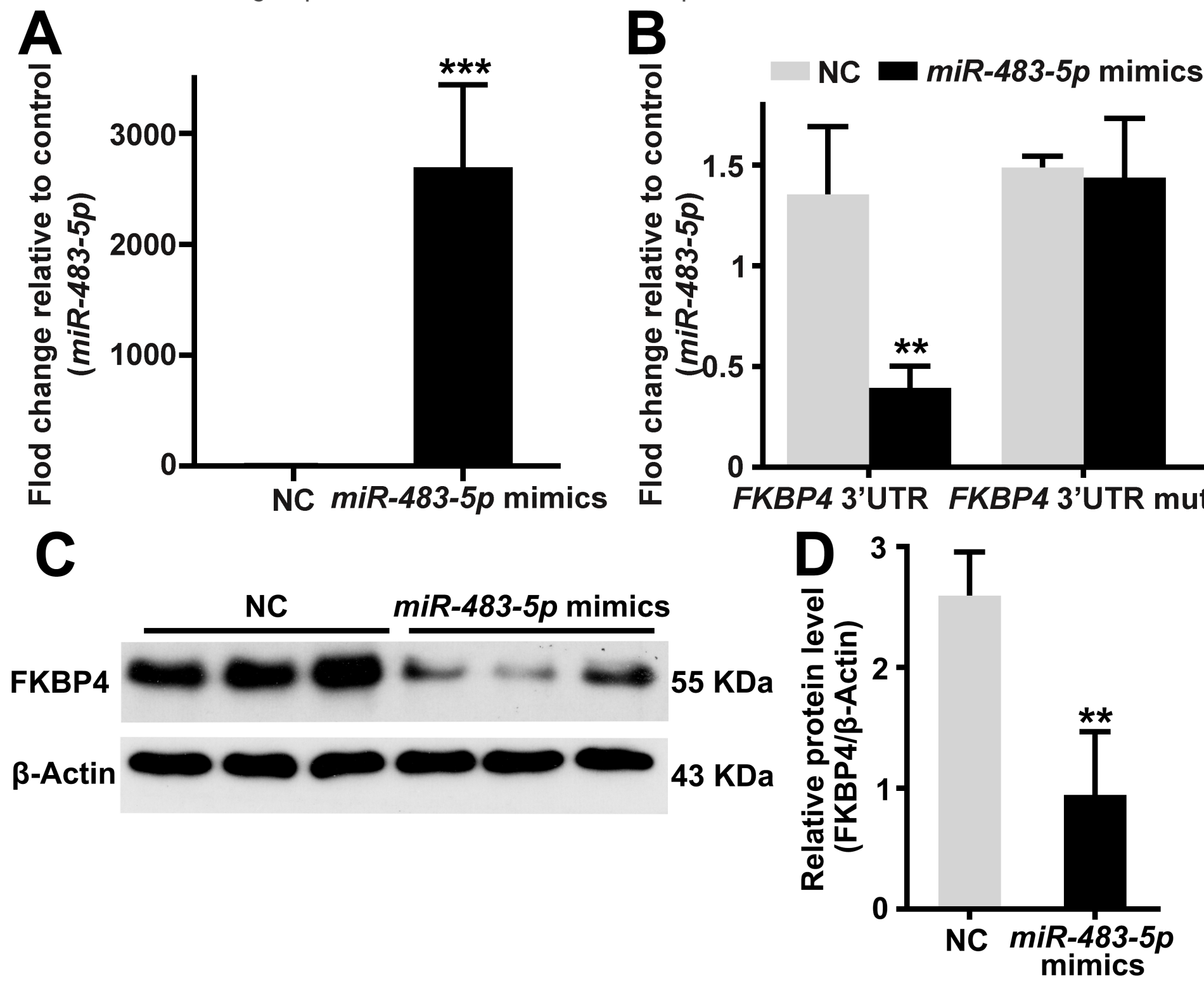

Figure 3 
FKBP4 is a functional target of miR-483-5pin vitro. a qRT-RCR analysis was used to test the transfection efficiency of miR-483-5p mimics in HeLa cells. b The specific regulation of FKBP4 by miR-483-5p was demonstrated using luciferase analysis. c The expression of FKBP4 protein was determined in HeLa cells transfected with miR-483-5p mimics by western blotting. $d$ Quantification analysis was normalized to that of $\beta$-Actin. NC, negative control. All data are shown as mean \pm SEM. $n=5$ for each group. ${ }^{\star \star} P<0.01$, *** $\mathrm{P}<0.001$, compared with controls.

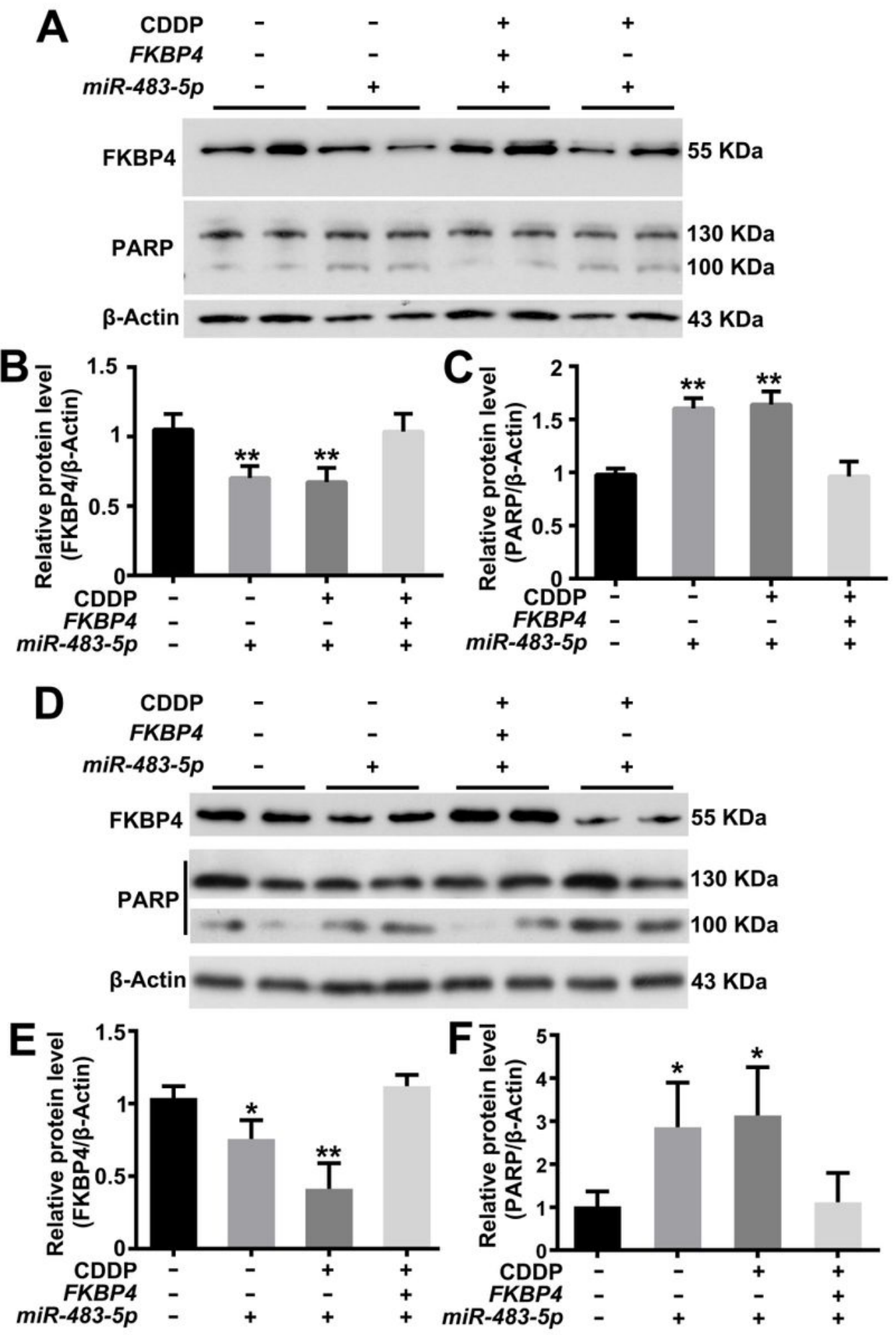

Figure 4 
Overexpression of FKBP4 in KGN and primary GCs could alleviate the apoptosis caused by CDDP plus miR-483-5p overexpression. a The expression levels of FKBP4 and PARP were determined in KGN treated by CDDP plus miR-483-5p or/and FKBP4 plasmid transfection using western blotting. Semi-quantification analysis of $b$ FKBP4 and $c$ PARP was normalized to that of $\beta$-Actin. $d$ The expression levels of FKBP4 and PARP were determined in primary GCs treated by CDDP plus miR-483-5p or/and FKBP4 plasmid transfection using western blotting. Semi-quantification analysis of e FKBP4 and $\mathrm{f}$ PARP was normalized to that of $\beta$-Actin. All data are shown as mean \pm SEM. $n=5$ for each group. * $P<0.05,{ }^{\star} P<0.01$, compared with controls. 


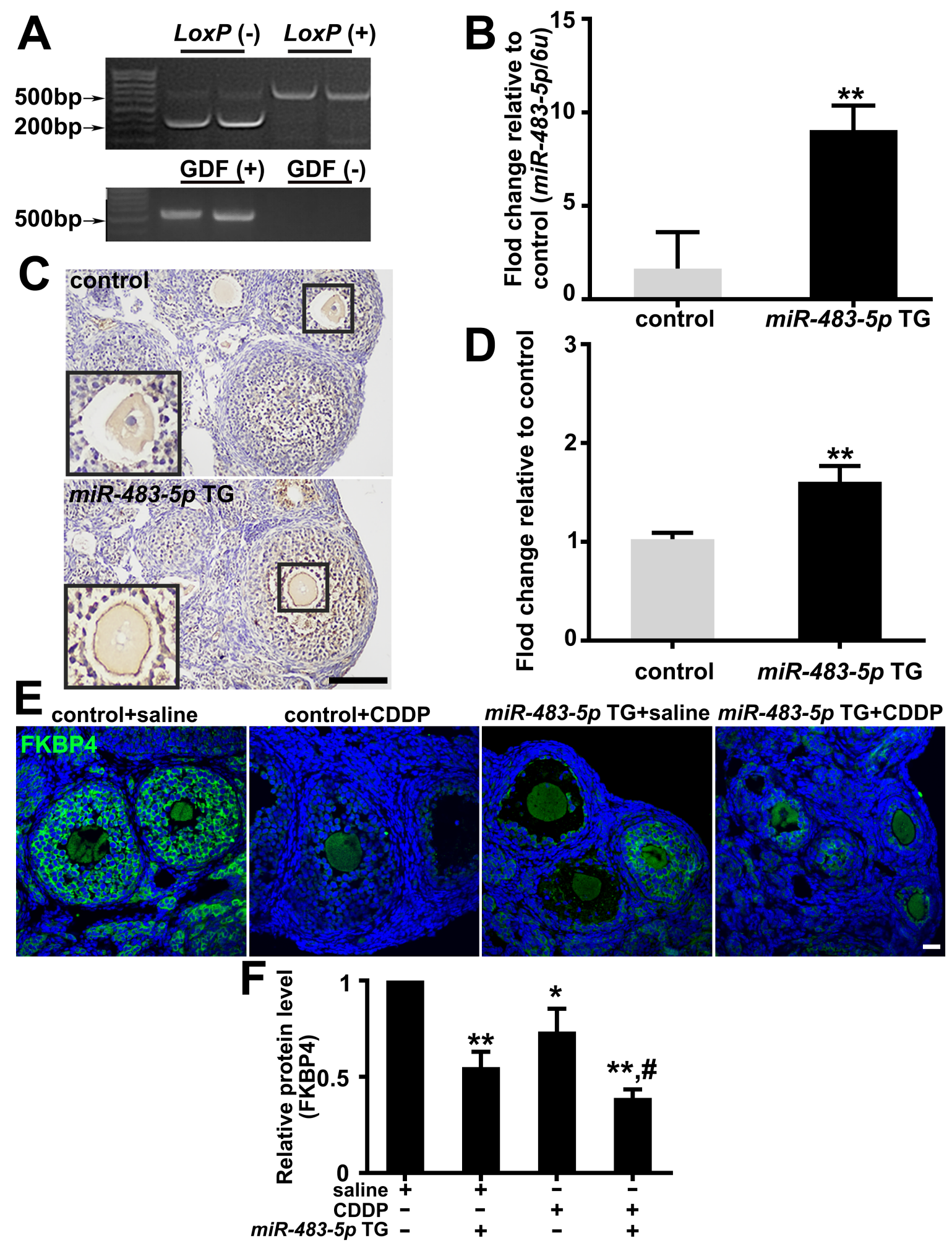

Figure 5

miR-483-5p TG mice were established using the Cre/LoxP system. Genotyping of F1 offspring for miR483-5p TG was carried out after mating transgenic Gdf 9-Cre and miR-483-5p LoxP mice. miR-483-5p TG was tested using a PCR and b qRT-PCR. The $200 \mathrm{bp}$ bands corresponds to the miR-483-5p LoxP(-), while the 500 bp band corresponds to miR-483-5p LoxP(+), and the band below the 500 bp band corresponds to Gdf9-Cre. The location and expression of miR-483-5p were tested in the ovaries from miR-483-5p TG 
mice using $c$ hybridization in situand $d$ semi-quantification analysis. The images shown in the lower left represent an enlarged view of the inserted frame. The location and expression of FKBP4 were tested in the ovaries from miR-483-5p TG mice using e immunofluorescence and f semi-quantification analysis.

The mouse model was divided into four groups, including control plus saline, control plus CDDP, miR-483$5 p$ TG plus saline, and miR-483-5p TG plus CDDP. All data are shown as mean \pm SEM. Scale bar $=50 \mu \mathrm{m}$. * $\mathrm{P}<0.05$, ** $\mathrm{P}<0.01$, compared with controls, $\# \mathrm{P}<0.01$, compared with CDDP.

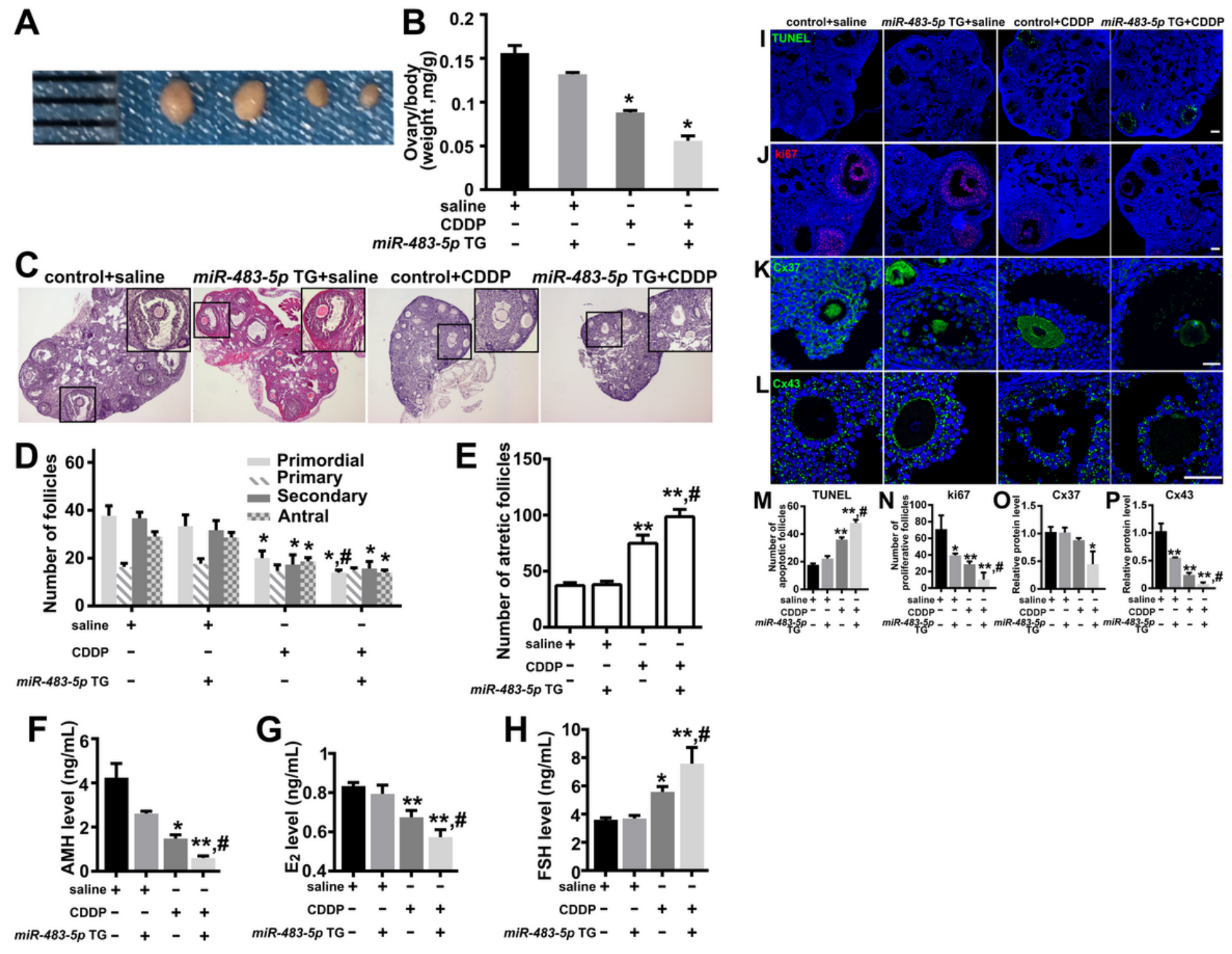

\section{Figure 6}

The overexpression of miR-483-5p in oocytes could enhance ovarian injury and sex hormone disturbances. Ovary a size and b weight were measured. c H\&E staining was used to investigate ovarian follicular development. Images in the upper right represent an enlarged view of the inserted frame. Ovarian follicle counts, including d primordial, primary, secondary and antral follicles and e atretic follicles were determined. Sex hormone levels, including $\mathrm{f} \mathrm{AMH,} \mathrm{g} \mathrm{E2,} \mathrm{and} \mathrm{h} \mathrm{FSH,} \mathrm{were} \mathrm{tested} \mathrm{in} \mathrm{the} \mathrm{serum}$ using ELISA. TUNEL and semi-quantification analysis were used to determine the proportion of $\mathrm{i}, \mathrm{m}$ apoptotic granulosa cells. Immunofluorescence and semi-quantification analysis were used to 
demonstrate the expression levels of $\mathrm{j}, \mathrm{n}$ ki67, $\mathrm{k}$, o Cx37and I, p Cx43. The mouse model was divided into four groups, including control plus saline, miR-483-5p TG plus saline, control plus CDDP, and miR-483-5p TG plus CDDP. All data are shown as mean \pm SEM. $n=5$ for each group. Scale bar $=50 \mu \mathrm{m}$. ${ }^{*} P<0.05$, ** $P$ $<0.01$, compared with controls, \# $\mathrm{P}<0.05, \mathrm{P}<0.01$, compared with the CDDP group.

\section{Supplementary Files}

This is a list of supplementary files associated with this preprint. Click to download.

- Figures1..tif 